\title{
IR Temperature Measurements to Determine Fin Effectiveness of Longitudinal Fins:
}

\author{
by A. Willockx*, C. T'Joen*, H.-J. Steeman*, H. Canière* and M. De Paepe*
}

${ }^{*}$ Department of Flow, Heat and Combustion Mechanics, Ghent University-UGENT, Gent, Belgium

\section{Introduction}

Fins are widely used in heat exchangers, for cooling of electronics,... A good fin performance indicator is important for the design. The concept fin effectiveness is a better performance indicator than fin efficiency, which is the commonly used parameter. The big disadvantage of fin efficiency is that the real performance of the fin is compared to an ideal non-existing fin of the same shape but with infinitely high thermal conductance. So fin efficiency is an idealization and has physically no meaning. This makes it difficult to compare the thermal performance of different fin forms. On the other hand, fin effectiveness has the advantage of measuring the heat transfer gain obtained by placement of the fin and gives an idea of its real performance. It gives the ratio of the heat transferred by the fin to the heat transferred from the surface covered by the fin's base under the same thermal conditions, in the absence of the fin. Fin effectiveness makes it possible to compare the fin quality of different types of fins. This can be used to develop a decision system to choose a fin type in order to remove a certain amount of heat from a surface for a given free surface area. The objective of this paper is to identify the fin effectiveness as well as the variation of the local convective heat transfer coefficient. Therefore the heat fluxes through the fin model and the primary surface need to be known. It is very difficult to do accurate heat flux measurements. It is easier and more common to measure surface temperatures.

\section{Experimental setup}

A test rig is designed in which both fin effectiveness and local heat transfer coefficients for different fin forms can be determined. In order to experimentally determine fin effectiveness, a primary surface has to be heated and the heat flux through this surface has to be known. In a second phase, a fin is placed on this primary surface and the heat flux through the fin also has to be determined. The setup is built as follows. A rectangular aluminum reference block is heated at the bottom. The upper surface of the block is the primary surface. A second aluminum block of the same dimensions is made, but with an aluminum cooling fin on top of it. Aluminum is used because of its high thermal conductivity $(k=200 \mathrm{~W} / \mathrm{mK}$.) A flexible isoflux heat foil is placed at the bottom of the reference block in order to induce a heat flux through the primary surface. The heat losses sideways and especially downwards need to be minimized in order to force all heat flux upwards through the aluminum block and fin. Therefore a guard heater assembly is constructed around the primary surface. The guard heater is set at the same temperature as the bottom and side walls of the aluminum block, which are measured with thermocouples. So there are almost no conduction heat losses at the bottom of the upper heat foil and from the side walls of the block due to a lack of temperature difference. The aluminum block with cooling fin and the guard heater assembly are insetted in a slab of polyurethane and this is placed in a wind tunnel with a rectangular test section (Fig. 1). The wind tunnel is used to examine the influence of the Re-number on the local convective heat transfer coefficient and the fin effectiveness. The Reynolds number (based on fin length as reference length) can be varied from 12750 to 63750 . The base of the fin is at the same height as the bottom of the wind tunnel to avoid disturbance of the air flow at the test section.

The temperature profiles on the fin surface and top of the reference block (primary surface) are measured by infrared thermography with a Midas infrared camera It is a long wave (LW) camera which has a spectral response from $8 \mu \mathrm{m}$ to $14 \mu \mathrm{m}$. Thermography has the big advantage that it is a non-intrusive measurement technique and a complete temperature distribution is obtained with one measurement. The front and back of the wind tunnel are made out of Plexiglas in order to see the test section. Because Plexiglas is not transparent for infrared radiation, it is impossible to measure the fin temperature with the infrared camera placed outside the wind tunnel. Therefore a small window made out of mica foil is placed in the side wall and top wall of the test section (Figure 1). The mica foil has a relatively high transmittance for IR-radiation in the LW spectrum. The temperature image of the front side can also be used for the rear side due to the symmetry of the setup and the isoflux heat flux at the bottom of the

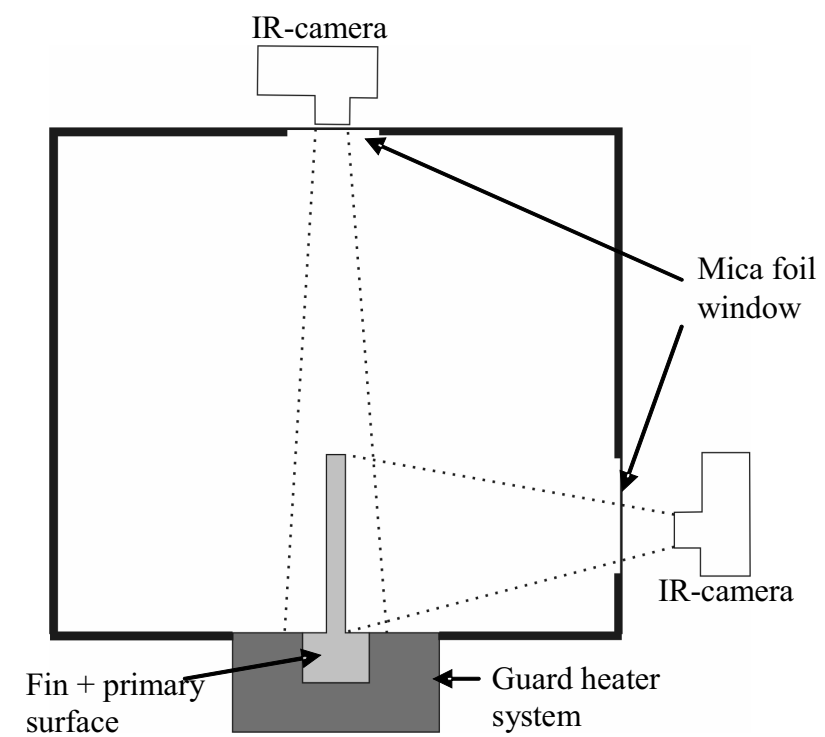

Fig.1: Experimental setup in test section wind tunnel 
block. On top of the wind tunnel, another mica foil window is placed to measure the temperatures of the upper side of the fin and the primary surface (Figure 1).

\subsection{Calibration IR-camera}

Three important parameters need to be calibrated in order to do accurate measurements: emissivity $\varepsilon$ of the the fin, transmittance $\tau$ the mica foil window and the parameter: $T_{\text {amb }}$. The fin and block are painted with matt black paint, which has a high emissivity. The emissivity is determined according to the ASTM Standard E 1933-99a method. An almost constant emissivity of $\varepsilon=0.929$ was measured in the range of measured fin temperatures $\left(40^{\circ} \mathrm{C}-80^{\circ} \mathrm{C}\right)$. The transmittance $\tau$ of the mica foil window is determined at $\tau=0.82$. The sensor is also exposed to radiation which consists of various environmental contributions like radiation from the wind tunnel walls or other elements outside the wind tunnel. These factors are gathered in one parameter: $T_{\text {amb. }}$. The influence of a small variation of this factor is negligible, mainly due to the high emissivity of the black paint. These three parameters are put in the camera as calibration values.

\subsection{Measurement data}

An example of the obtained temperature measurement data is shown in figure 2 for a longitudinal plate fin. In these infrared images, each pixel represents a temperature measurement.

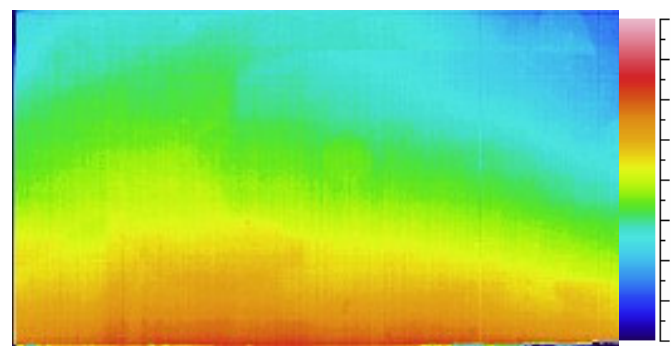

a. Front view of fin

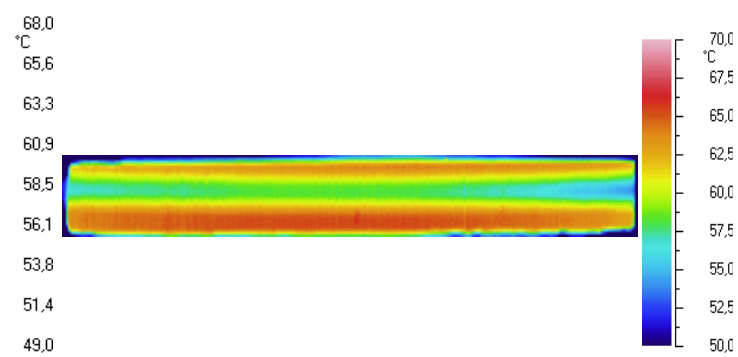

b. Top view of fin

Fig. 2: Infrared temperature measurement of a longitudinal plate fin

\section{Inverse conduction problem}

These temperature measurement data are used as boundary conditions for a $3 \mathrm{D}$ numerical model of the fin and the primary surface. In order to determine the heat fluxes through the cooling fin from these temperature measurements, an inverse heat conduction problem with the following boundary conditions has to be solved: an imposed heat flux at the bottom wall of the primary surface and measured temperature profiles at the fin walls and upper walls of the primary surface. Inverse conduction problems are typically ill-posed and cannot be solved directly. Therefore an optimization method is used to find a solution for the local heat fluxes on the walls where the temperatures are measured, so that the error between the measured and calculated temperature values drops under a critical value. A numerical algorithm based on the conjugate gradient method is set up to solve this three-dimensional inverse problem. However, the heat fluxes on the fin surface obtained with this numerical algorithm still show significant fluctuations which are not physical but inherent to ill-posed problems. In order to dampen these fluctuations, a first order Tikhonov regularization is added to the numerical algorithm.

\section{Results}

As a result the variation of local convection coefficients over the fin walls are obtained. This makes it possible to determine fin effectiveness and to observe the local effects that influence the heat transfer and fin effectiveness. The fin effectiveness of a longitudinal plate fin is plotted in figure 3 for different Re-numbers.

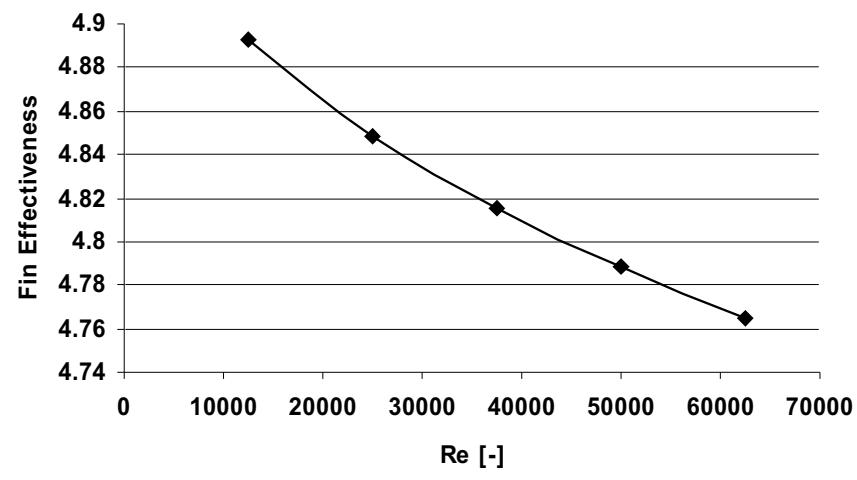

Fig. 3: Fin effectiveness at different Re-numbers for a straight plate fin 